\title{
Image guided reconstruction of un-sampled data: a coherent filling for uncomplete Cultural Heritage models
}

\author{
Matteo Dellepiane \\ ISTI-CNR \\ Via Moruzzi 1, 56124 \\ Pisa (PI) Italy \\ dellepianedisti.cnr.it
}

\author{
Andrea Venturi \\ ISTI-CNR \\ Via Moruzzi 1, 56124 \\ Pisa (PI) Italy \\ andrea.venturi@gmail.com
}

\author{
Roberto Scopigno \\ ISTI-CNR \\ Via Moruzzi 1, 56124 \\ Pisa (PI) Italy \\ scopignodisti.cnr.it
}

\begin{abstract}
Nowadays, 3D scanning is widely applied to Cultural Heritage applications. In this peculiar context, the fact that the 3D models obtained from scanning are usually uncomplete can be an issue, especially because automatic hole filling techniques can create inaccurate surfaces.

In this paper, we present a method to reconstruct unsampled portions of the $3 D$ models by inferring information about the real shape of the missing part from an image. The needed data for surface creation are extracted from a predefined pattern which is projected on the real object, in the zone where the geometry of the $3 D$ model is missing.

The procedure, which is almost completely automatic, analyzes the image in order to extract the pattern and estimate the projector position. Then, the extracted information is used to obtain a hole filling which is coherent with the real shape of the object. A series of test on real objects proves that our method is able to recover geometrical features that cannot be reconstructed using state-of-the-art methods. Consequently, it can be used to obtain complete $3 D$ models without creating false data.
\end{abstract}

\section{Introduction}

The last few years of technological development made 3D Scanning a mature technology. This is a consequence not only of the improvements of the acquisition devices, but also of the development of new algorithms which automatize most of the required raw data processing and fully exploit the resources of graphics hardware.

One of the most important fields of application of 3D scanning is Cultural Heritage. There are several reasons behind this: the sampling accuracy and speed granted by this technology, the variety of different objects that can be acquired, the possibility to acquire data without touching the objects, the wide number of possible uses of the digital 3D models (from archival and restoration support to rapid prototyping). While 3D scanning is able to provide an extremely accurate representation of an object, the final digital 3D model can suffer from a number of geometric artifacts (singular vertices, overlaps/folds, self-intersections, complex edges, holes). Some of them can be generated during the acquisition of the single range maps, while others are introduced during the processing of the raw data.

Artifacts removal is a quite important subject in the Computer Graphics and Computer Vision research: while some of the artifacts types can be easily detected and removed, others can present difficulties in both detection and removal. Holes are a common and unavoidable artifact in scanned meshes. 3D models obtained via 3D Scanning are very rarely complete, since usually not all the parts of an object can be reached by the scanner. Moreover, it is also possible that some parts are not covered due to errors or carelessness during the acquisition campaign.

The presence of holes in a 3D model can be distracting and annoying during visualization, hence several methods to automatically fill the holes were presented in literature, including reconstruction methods that produce water-tight models. Most of these approaches rely on the analysis of the existing geometry of the model: even in the case of holes of medium and large size, the new geometry can be almost indistinguishable from the rest of the model.

Nevertheless, the hole filling operation can be a problem in the case of Cultural Heritage, since the geometry created is a plausible but essentially guessed information. On the contrary, the approach of Cultural Heritage experts usually tries to avoid the creation of not reliable data. Hence, the final geometry is usually kept uncomplete unless the hole filling is key for further applications (e.g. rapid prototyping).

In this paper, we present an approach that aims to fill the holes of an uncomplete mesh by extracting information about the missing geometry from one or more images. The data are obtained by analyzing images of the real object, on 

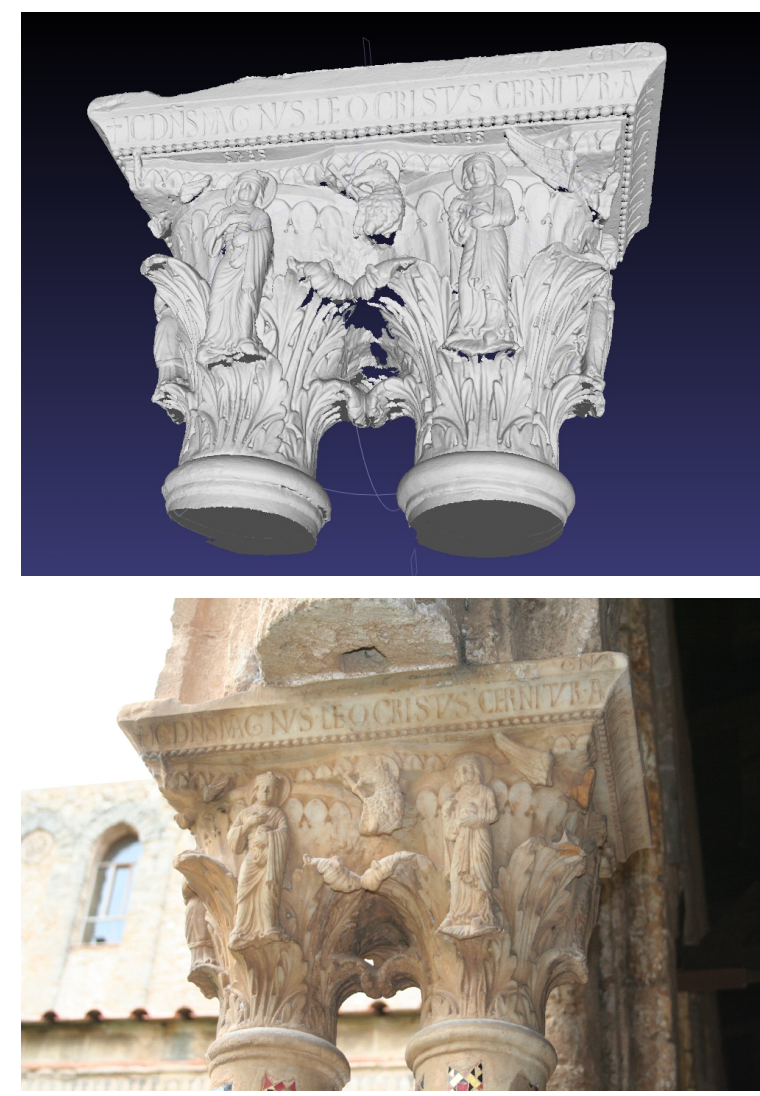

Figure 1. A rendering of an uncomplete model, where part of the geometry could not be acquired with laser scanner, and an image which depicts the missing zones.

which a pre-defined colored pattern (generated by a projector or a laser diode) is projected on the zone which was not covered by the scanner. Using a digital camera, it is much easier to frame partially occluded regions: an example of an uncomplete model is presented in Figure 1, where a number of small regions of the capital were not be sampled properly (in most cases, since they were hidden either to the emitter or to the sensor of the triangulation-based scanning device). The same regions can be easily framed using a digital camera. Nevertheless, without further information it can be hard to infer data about the geometry of the object:. Conversely, the analysis of the shape of the projected pattern gives additional information that can be used to find a shape-congruent patch to close the hole.

In our approach, the hole patch is obtained with a semiautomatic process: the first step is to align the image on the $3 \mathrm{D}$ model (this is the only manual step in our current implementation, but automatic solutions are possible also for this phase); then, the pattern is extracted from the image and, given the knowledge of the calibrated projector position wrt. the camera parameters, we are able to estimate the geometry of several points lying on the object surface and corresponding to the reflected pattern; these geometric data are then used, together with the hole open border and the nearby surface to reconstruct the patching sub-mesh. In this way, even the geometric features which were completely lost due to the lack of information can be recovered.

The paper is organized as follows. An overview of the hole filling issues and previous solutions is presented in Section 2. The requirements and solutions for the image acquisition setup are discussed in Section 3, while Section 4 describes the hole filling process. Some results and a comparison with state-of-the-art methods are shown in Section 5. Finally, conclusions and future work are presented in Section 6.

\section{Related Work}

Hole filling has been an important field of research in recent years. Closing holes of small size is a trivial operation. Unfortunately, 3D scanning produces many holes (usually from hundreds to thousands in the case of complex models) and many of them have a large extension, a high number of border edges and a complex shape. Examples of the complexity of the possible holes are presented in [6]. In this case, the filling operation can be very long and prone to errors.

Most of the proposed approach assume no information about the missing geometry. Hence, this information is mainly extracted from the shape of the model around the hole. These hole filling techniques can be roughly divided in two groups: volumetric and surface oriented.

In the context of the volumetric techniques, the model or the sampled data are usually immersed in a volumetric grid and all the holes are filled while reconstructing the entire shape. These methods essentially use regular $[13,8,6]$ or adaptive [4] grids. They are usually quite robust, able to deal with isles and high resolution models, since they essentially derive from the Marching Cubes approach [18]. Nevertheless, there is a substantial remeshing of the geometry, and all the holes are filled (they cannot be managed selectively). These modifications could not be wanted by the user.

The surface oriented algorithms work on a single hole at a time, and the new information is usually inferred by analyzing the shape of the surface near the border of the hole. A simple but effective method was proposed by Varnuška et al. [15]. Other approaches use Radial Basis Functions [5] or Moving Least Squares [2] to obtain a smooth filling surface. Another example is the method proposed by Liepa [10], that is able to provide very good results. It is divided in three main phases: in the first one, a rough triangulation that fills the hole is found, the the remeshing and smoothing phases produce an almost undistinguishable new surface.

Another subgroup of surface oriented approaches extract information from the analysis of all the geometry of the model. Bendels [3] and Sharf [17] extend the idea of image inpainting and texture synthesis by analyzing the model 
and finding surfaces whose shape is similar to the ones near the border of the hole. Then, the hole is filled using patches of already existing geometry. Park et al [14] use a similar idea to reconstruct both shape and appearance from scanned data.

Surface oriented approaches can obtain good results and act selectively on single holes. Unfortunately, the complexity of the algorithm can be very high when the number of border edges increases.

As already stated, the methods cited until now don't assume knowledge of the real shape the missing surface. An alternative idea is to infer missing information from an external source: Akbarzadeh et al [1] combine a large set of different data to complete a model of a city landscape. Analogously, it is possible to use data obtained from photogrammetry [16] to fill the holes in architectural models. Nevertheless, the acquisition of these integration data can be quite cumbersome.

$\mathrm{Xu}$ [19] proposed a much simpler source of data: a single image of the object. It is used to estimate a set of normal vectors, which generates a smooth surface which should recover the real shape of the object. Unfortunately, the results shown in the paper are obtained using renderings of an uncomplete model. No example using real images is shown.

\section{The acquisition setup}

The aim of the proposed work was to provide a method which should be able to fill the holes of a 3D model using information about the real shape of the missing part. This is a key issue for the use of such an algorithm in the context of Cultural Heritage (but also in other important application fields, like industrial prototyping).

Moreover, to allow easy deployment for $\mathrm{CH}$ applications, we decided to use the simplest possible type of additional data. Clearly, digital images are a very easy-to-obtain and flexible source. Acquiring an image is a very fast operation, and a large amount of data is obtained. Moreover, the easy handling of a digital cameras allows to frame portions of an object which cannot be easily reached by a scanner. Unfortunately, as the results of the work of Xu [19] showed, the processing of an image is still an hard task, especially when it's necessary to discriminate between geometric and appearance information. For this reason, it was decided to project a pre-defined pattern on the real object, and to analyze its shape on the image in order to infer data about the surface that has to be reconstructed. Accurate patterns can be generated even by low-end laser diodes: hence, it is possible to think of a compact and cheap acquisition device formed by a digital camera and a laser diode mounted on it. With this device, the user could take photos of the parts of the object which were not covered by the scanner, and then the images can be used to complete the 3D model. In the next Sections, we also used a projector to generate the patterns, but all the processing pipeline is clearly independent of the type of pattern emitter adopted.

In a more generic fashion, our acquisition setup is composed by a digital camera and a pattern projector whose position is not coincident with the one of the camera (to avoid parallax effect). Even though also in this case some part could not be reached due to the different positions of emitter and objective, the advantage of this device would be the much higher handiness. The example in Figure 1 shows that digital cameras can easily frame portions that are unreachable for a 3D scanner.

A further choice about the acquisition device is the type of pattern to project on the model. Three examples are shown in Figure 2. It was decided to consider only patterns which are inscribed in a pre-defined shape (like the square in the central and righthand examples of Figure 2). This was extremely important for the definition and robustness of the image processing and projector position estimation steps (see next Section).

The output of the acquisition setup is one or more images of the object on which a (pre-defined) pattern is projected. The necessary steps to extract information which will be used to fill the hole are presented in next Section.

\section{Data processing and hole filling}

The data processing pipeline needed to fill the holes starting from the image can be divided in the following steps:

- For each image framing the hole region:

- image registration on the 3D model (image-togeometry transformation);

- extraction of the pattern pixels from the image;

- estimation of the position and orientation of the pattern projector;

- Hole filling, using the knowledge gained with the projected pattern points.

All of the steps are either automatic or semi-automatic. The whole filling operation is completed within minutes, taking into account both user intervention and processing time. The next subsection will present each processing step and the intermediate data extracted.

\subsection{Image-to-geometry alignment}

In order to be able to reconstruct the missing geometry, it is necessary to estimate the the position of the elements of the acquisition setup. The first step is to find the position of the digital camera wrt. the sampled artifact. This is 

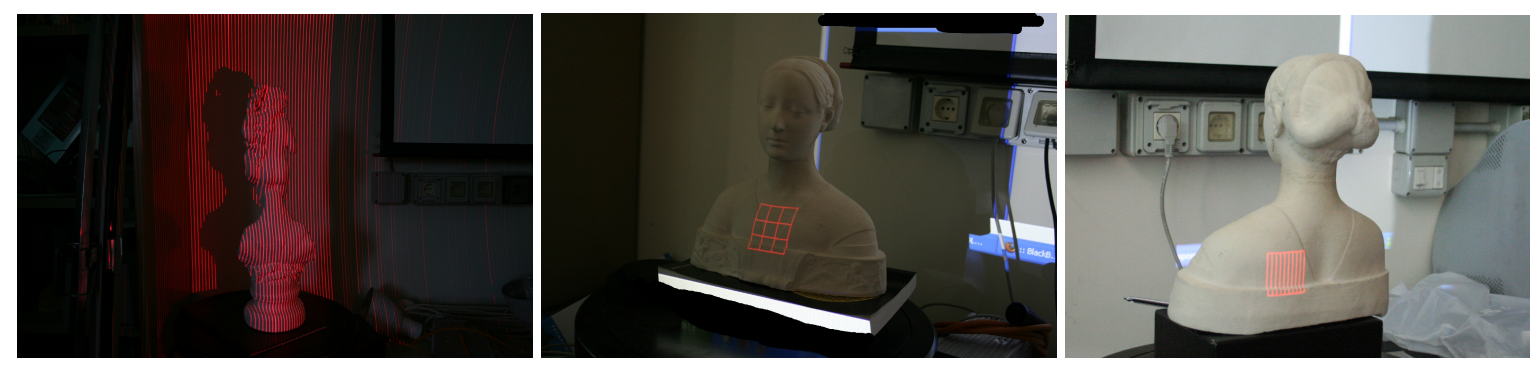

Figure 2. Three examples of possible projected patterns.
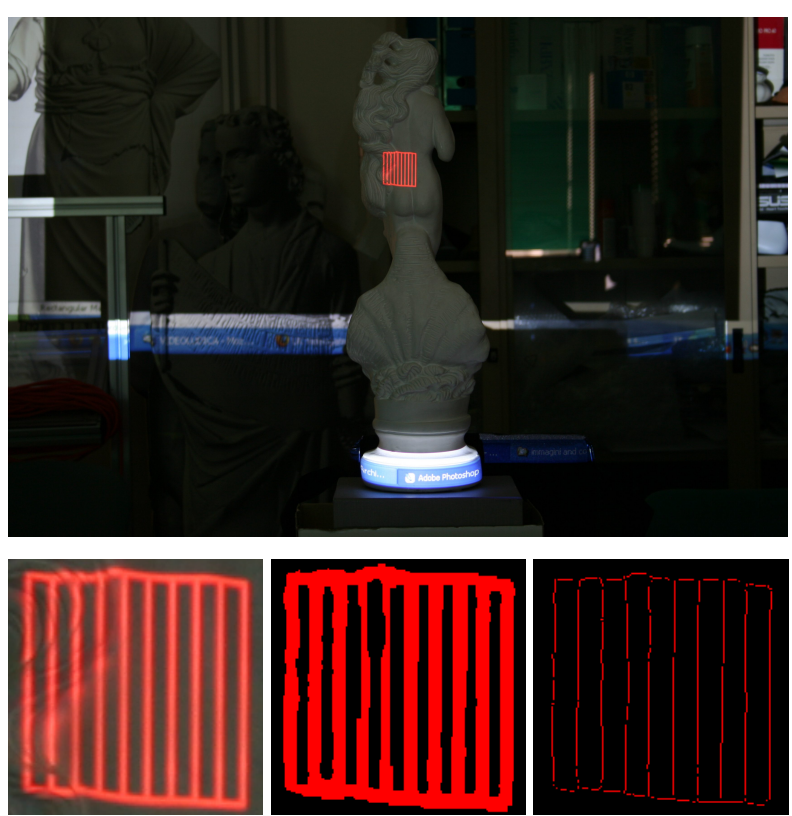

Figure 3. An example of pattern extraction from an image. Top: the original image. Left: the portion of the original image depicting the pattern. Center: the extracted red channel. Right: the extracted pattern after the application of the erosion filter.

obtained by registering the image to the digital 3D model.

There are several automatic and semi-automatic methods to do this $[9,11]$. In our paper, we used a semi-automatic approach [7] which allows to obtain the estimation of the camera parameters (intrinsics and estrinsics) in a couple of minutes. The knowledge of the camera position is critical in order to be able to estimate the projector position and to transform the extracted 2D information (pattern pixels location) into points in 3D space .

\subsection{Pattern extraction from image}

In addition to the camera position, it is necessary to analyze the image in order to extract further information about the projected pattern. This is done by applying some common image processing filters to find and extract the pattern.

The first operation is to find the pattern pixels in the con- text of the image. Although this can be done automatically, in the current implementation the user is asked to pick the four external corners of the pattern on the image. These user-defined data are also used to enhance the projector position estimation. Once that the user has selected the four corners, the bounding square of the pattern is extracted (see Figure 3-left).

Then, the further processing is operated only on the red channel (Figure 3-center). Since we want to associate each line of a pattern to a single line of pixels in the image, an erosion filter is applied in order to extract the central line of pixels for each line of the pattern (Figure 3-right).

\subsection{Pattern projector position estimation}

One possible design for the acquisition device, as described in Section 3, is to couple a digital camera with a small projector (e.g. a laser diode) firmly mounted on it. In this case, the calibration of the device should be done only once in its lifetime (for example, by taking pictures of a calibration object and calculating the relative distance between the camera and the projector). Known the camera-projector calibration and the image-to-geometry alignment to the 3D model (see previous subsection), the position of the projector can be automatically computed.

Nevertheless, we decided to take into account a more general case, where the projector position is not known in advance. The estimation of the projector position is a key element for the accuracy of the overall geometry reconstruction process. The data extracted in the previous steps are used in order to perform it. Moreover, it's necessary to have a model of the pattern (essentially the divergence angle of the projected grid). In the case of a laser diode, it can be inferred from the specifications, otherwise it can be easily calculated by measuring the size of the projected grid at different known distances.

The projector position is estimated using an implementation [12] of the Levenberg Marquardt method, which is a robust MSE estimator for nonlinear problems. The input data to the solver are the model of the projector and a number (16 in our tests) of points chosen from the external lines of the pattern extracted from the image (see previous subsection). The pattern pixels on the image are projected 
on the 3D model (using the camera data obtained from the image-to-geometry registration phase). Then, the algorithm iteratively fits the projector model to the set of $3 \mathrm{D}$ points until convergence.

This method proved to be quite robust and fast, since convergence is usually obtained in seconds; its accuracy was assessed with the analysis of the results presented in the next section. As a matter of fact, small errors in position estimation would generate visible inaccuracies in geometry reconstruction.

\subsection{Geometry reconstruction and hole filling}

The previous steps extracted from the image all the data which are necessary to drive the hole filling process. Knowing the camera and the projector positions and orientations, and the coordinates of the pixels of the image associated to the pattern, it is possible to apply triangulation (in the same fashion as the laser and structured light triangulation $3 \mathrm{D}$ scanners) in order to associate a $3 \mathrm{D}$ position to each pattern pixel of the image.

Hence, a new 3D point can be created for each pattern pixel which maps on the hole zone. Before starting geometry reconstruction, we take advantage of the redundancy of data in order to improve the accuracy. In a first stage, only the pixels which map on the already existing geometry of the model are taken into account. For each pixel, the 3D coordinates obtained via triangulation and the projection of the pixel on the 3D model are compared. The value of the differences in depth of the two positions (averaged over all the considered pixels) is used as a correction factor that takes into account the possible inaccuracy introduced in the projector position estimation phase.

Finally, the reconstruction of the surface can start. For each pattern pixel not projecting on the 3D model, a 3D position is calculated (taking into account also the correction factor) - Starting from the new 3D points, several strips of new triangles are created (see Figure 4a) and then connected to the hole borders. This is the key step in the whole procedure: the hole filling problem is split in several simpler hole filling problems. But the splitting is obtained by inserting information about the real shape of the object. In this way, eventual features which were completely absent can be (at least partially) retrieved.

After this very important stage, the hole filling algorithm is very similar to the one proposed by Liepa [10]: in a first stage, a rough filling is obtained by finding minimum-area spanning surface which also takes into account the dihedral angles between adjacent triangles (4b). Then, a remeshing phase splits the newly created triangles until their average size is similar to the one of the rest of the model (4c). Finally, a smoothing filter makes the new surface almost undistinguishable from the rest of the model (4d).
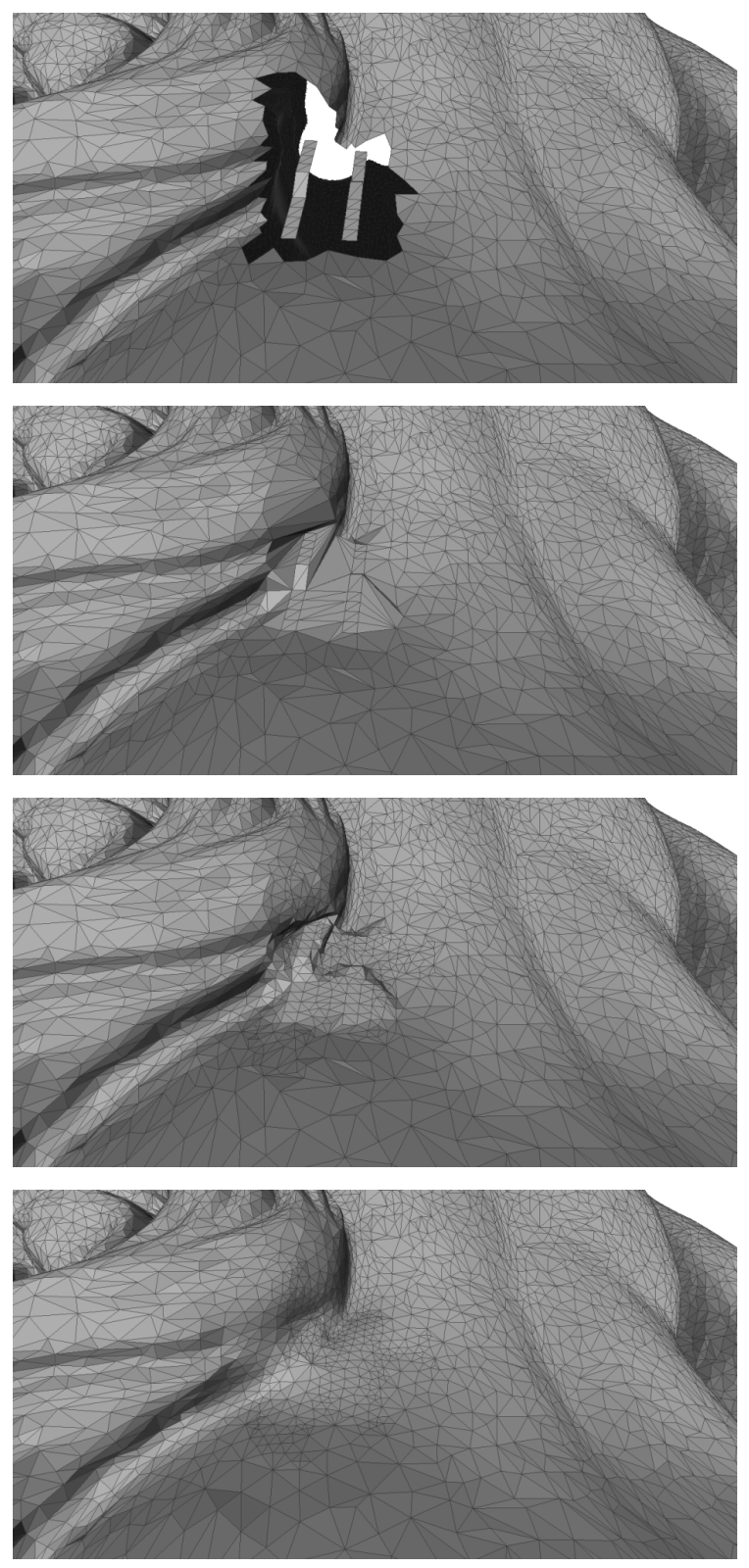

Figure 4. The hole filling procedure. From top to bottom: (a) the hole with the new triangle strips, (b) rough triangulation, (c) remeshing, (d) smoothing

In conclusion, once that the input data have been analyzed, the hole filling operation is completely automatic, and the quality of the results is extremely good.

\section{Results and comparison}

The previous Section showed that the quality of the reconstructed surface is comparable to the results obtained from the state-of-the-art hole filling methods. But the main goal of the method was to be able to recover geometric features that couldn't be reconstructed without some knowl- 


\begin{tabular}{|c|c|c|c|}
\hline Algorithm & Average & Maximum & RMSE \\
\hline Test 1 & & & \\
\hline Liepa & 0,002083 & 3,284013 & 0,052673 \\
\hline EarCut & 0,000593 & 0,687241 & 0,01462 \\
\hline Our method & 0,001201 & 1,708783 & 0,0295663 \\
\hline Test 2 & & & \\
\hline Liepa & 0,515288 & 33,177967 & 3,379488 \\
\hline EarCut & 0,000553 & 0,79688 & 0,015343 \\
\hline Our method & 0,000222 & 0,642542 & 0,007969 \\
\hline Test 3 & & & \\
\hline Liepa & 0,497988 & 33,177967 & 3,32634 \\
\hline EarCut & 0,00122 & 1,67301 & 0,027455 \\
\hline Our method & 0,000405 & 1,048663 & 0,013196 \\
\hline Test 4 & & & \\
\hline Liepa & 0,002006 & 3,284013 & 0,051815 \\
\hline EarCut & 0,000294 & 0,485467 & 0,008804 \\
\hline Our method & 0,000493 & 1,919416 & 0,018686 \\
\hline
\end{tabular}

Table 1. Analysis of Hausdorff distance between the original and the reconstructed models

edge of the shape of the object.

Hence, a set of test cases was created to compare the results of our method with other techniques, not only in terms of visual pleasantness, but especially in terms of geometric accuracy. In order to perform this, we created artificial holes in several complete models (acquired with 3D scanning technology) and, then, we compared the results obtained with our method and with two reference alternative methods: Earcut [15] and Liepa [10]. The first one is a very simple but effective method, where the final triangulation is usually quite rough. The second method, while more complex, provides visually pleasant fillings.

The tests were created in order to cover different possible cases, including the case where no particular geometric feature is missing and the one where important data are partially or totally missing. The results of two tests are shown in Figure 5. In the second one, a visible geometric feature is removed from the model: the result obtained with Leipa has a smooth shape, but information about the geometric features is totally lost. The same can be observed on the EarCut result, with the addition of a lower quality triangulation. Our method is instead able to partially recover the detail. In the last case, all methods obtain similar results.

Visual comparison is not enough to validate the accuracy of our method. For this reason, we calculated the Hausdorff distance between the original and the reconstructed models. This distance allows to measure the actual accuracy of the newly created geometries. The results of four test sets are shown in Table 1. The first observation is that the Liepa results are the less accurate ones: the error is introduced by the remeshing and smoothing phase, which improves the appearance of the surface, but introduces a quite high error. The triangulation obtained by EarCut is quite similar to the original mesh, but it is often visually unpleasant. Our method offers a very good compromise between accuracy and appearance: while producing smooth surfaces, the error is always comparable (if not lower) to the EarCut.

Considering also that our method shares the triangulation, remeshing and smoothing phase with Leipa, this further shows that, since almost no error is added, this is due to the fact that the information extracted from images is really a representation of the shape of the object. Otherwise, the Hausdorff distance value would be comparable.

\section{Discussion, future work and conclusions}

The presented system is a compact and easy to use way to close portions of surfaces which cannot be reached by laser scanner. It is mainly based on the triangulation measurement; hence, it suffers from the same weak-points of the triangulation devices. But most of them can be overcome by two main advantages: the framed area and the handiness. The fact that a much smaller area is framed and reconstructed reduces greatly the cone of occlusion. Moreover, the digital camera is much more handy than a scanner, hence most of the occlusion issues can be solved by moving or rotating it. Its handiness permits also to reach areas which cannot be reached by scanners, due to their size.

An alternative solution to this system could be the use of a Z-Cam, which calculates the depth of points in the space using time-of-flight. In this way, the problem of occlusion is eliminated. Unfortunately, the current precision of these devices (1-2 cm of error on the single measurement) and their cost make them still not usable in the practical case. The previous Section showed that the use of our simple acquisition setup can lead to a coherent completion of un-sampled geometry. In particular, it was proved that it is possible to extract accurate geometric information using a simple image. While for the testing part only one image at a time was used, it is possible to integrate the data coming for multiple images in order to improve accuracy in reconstruction.

Other possible future improvements can be found. First of all, considering the decrease in costs of laser diodes with custom patterns, the first goal is to build a low-cost device on which our system can be used in practice. This could lead to a more in-depth test on a set of more complex real cases.

Regarding the improvement of the processing part, the image processing can be made completely automatic, and more robust filter can be experimented in order to extract the pattern from the images. An alternative solution could be the use of a filter on the camera objective (like the ones 

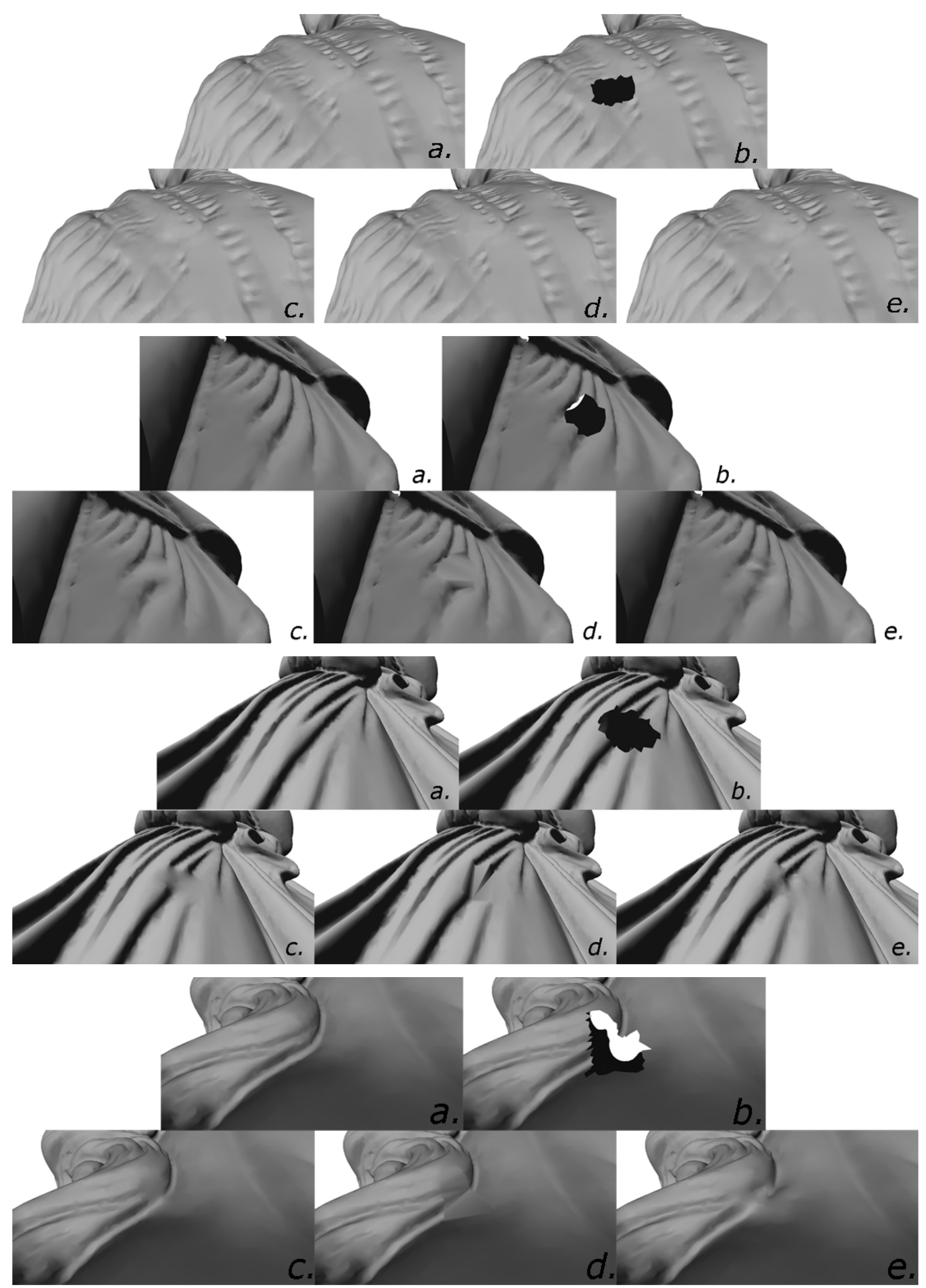

Figure 5. Four of the tests performed to validate our method. (a) the original mesh, (b) the hole created in the mesh, (c) Liepa [10] result, (d) EarCut [15] result, (e) our method result 
used by laser triangulation scanner) in order to further facilitate the pattern identification and extraction.

Finally, other applications can be found for this system. For example, the pattern can be used to improve the accuracy of model acquired with lower-accuracy devices (i.e. timeof-flight scanners). Once that an important detailed part is found, several images can be taken in order to improve the quality of the acquired mesh by deforming the geometry in order to fit the data extracted from the images.

In conclusion, a simple, semi-automatic and robust method to complete un-sampled data (especially in the field of Cultural Heritage) was presented. This system can guarantee the reliable completion of incomplete $3 \mathrm{D}$ model, so that they can be used for a wide range of applications, from mere visualization to rapid prototyping.

Acknowledgements This work was partially funded by EU IST IP 3DCOFORM and Tuscany Regional Project STArT. We would like to thank Paolo Cignoni for his supervision and Federico Ponchio and Massimiliano Corsini for their help.

\section{References}

[1] A. Akbarzadeh, J.-M. Frahm, P. Mordohai, B. Clipp, C. Engels, D. Gallup, P. Merrell, M. Phelps, S. Sinha, B. Talton, L. Wang, Q. Yang, H. Stewenius, R. Yang, G. Welch, H. Towles, D. Nister, and M. Pollefeys. Towards urban 3d reconstruction from video. In $3 D$ Data Processing, Visualization, and Transmission, Third International Symposium on, pages 1-8, June 2006.

[2] N. Amenta and Y. J. Kil. Defining point-set surfaces. ACM Trans. Graph., 23(3):264-270, 2004.

[3] G. H. Bendels, R. Schnabel, and R. Klein. Detail-preserving surface inpainting. In M. Mudge, N. Ryan, and R. Scopigno, editors, The 6th International Symposium on Virtual Reality, Archaeology and Cultural Heritage, pages 41-48, Pisa, Italy, 2005. Eurographics Association.

[4] S. Bischoff, D. Pavic, and L. Kobbelt. Automatic restoration of polygon models. ACM Trans. Graph., 24(4):1332-1352, 2005.

[5] J. C. Carr, R. K. Beatson, J. B. Cherrie, T. J. Mitchell, W. R. Fright, B. C. McCallum, and T. R. Evans. Reconstruction and representation of $3 \mathrm{~d}$ objects with radial basis functions. In SIGGRAPH, pages 67-76, 2001.

[6] J. Davis, S. Marshner, M. Garr, and M. Levoy. Filling holes in complex surfaces using volumetric diffusion. In First Int. Symp. on 3D Data Processing, Visualization and Transmission (3DPVT'02), pages 428-438. IEEE Comp. Soc., 2002.

[7] T. Franken, M. Dellepiane, F. Ganovelli, P. Cignoni, C. Montani, and R. Scopigno. Minimizing user intervention in registering 2D images to 3D models. The Visual Computer, 21(810):619-628, sep 2005.

[8] T. Ju. Robust repair of polygonal models. ACM Trans. Graph., 23(3):888-895, 2004.
[9] H. P. A. Lensch, W. Heidrich, and H.-P. Seidel. Automated texture registration and stitching for real world models. In PG '00: Proceedings of the 8th Pacific Conference on Computer Graphics and Applications, page 317, Washington, DC, USA, 2000. IEEE Computer Society.

[10] P. Liepa. Filling holes in meshes. In SGP '03: Proceedings of the 2003 Eurographics/ACM SIGGRAPH symposium on Geometry processing, pages 200-205, Aire-la-Ville, Switzerland, Switzerland, 2003. Eurographics Association.

[11] L. Liu, I. Stamos, G. Yu, G. Wolberg, and S. Zokai. Multiview geometry for texture mapping $2 \mathrm{~d}$ images onto $3 \mathrm{~d}$ range data. Computer Vision and Pattern Recognition, 02:22932300, 2006.

[12] M. Lourakis. levmar: Levenberg-marquardt nonlinear least squares algorithms in $\mathrm{C} / \mathrm{C}++$. [web page] http://www.ics.forth.gr/ lourakis/levmar/+, Jul. 2004.

[13] F. Nooruddin and G. Turk. Simplification and repair of polygonal models using volumetric techniques. IEEE Transactions on Visualization and Computer Graphics, 9(2):191205, 2003.

[14] S. Park, X. Guo, H. Shin, and H. Qin. Shape and appearance repair for incomplete point surfaces. In Computer Vision, 2005. ICCV 2005. Tenth IEEE International Conference on, volume 2, pages 1260-1267 Vol. 2, Oct. 2005.

[15] M. V. J. Parus and I. Kolingerová. Simple holes triangulation in surface reconstruction. In A. Handlovivèová, editor, Algoritmy 2005. Slovak University of Technology, mar 2005.

[16] H. Przybilla. Fusion of terrestrial laserscanning and digital photogrammetry. 2006.

[17] A. Sharf, M. Alexa, and D. Cohen-Or. Context-based surface completion. ACM Trans. Graph., 23(3):878-887, 2004.

[18] H. E. C. William E. Lorensen. Marching cubes: A high resolution 3d surface construction algorithm. Computer Graphics, 21(4), 1987.

[19] S. Xu, A. Georghiades, H. Rushmeier, J. Dorsey, and L. McMillan. Image guided geometry inference. In 3DPVT '06: Proceedings of the Third International Symposium on 3D Data Processing, Visualization, and Transmission (3DPVT'06), pages 310-317, Washington, DC, USA, 2006. IEEE Computer Society. 